\title{
Alkali N.M.R. experiments on the radical ion pairs of biphenyl and fluorenone
}

\section{Part I. Analysis of N.M.R. shifts}

\author{
by G. W. CANTERS $†$ and E. DE BOER
}

Department of Physical Chemistry, University of Nijmegen, The Netherlands

(Received 12 February 1973)

\begin{abstract}
Results of ${ }^{6} \mathrm{Li},{ }^{7} \mathrm{Li},{ }^{28} \mathrm{Na},{ }^{99} \mathrm{~K},{ }^{85} \mathrm{Rb},{ }^{87} \mathrm{Rb}$ and ${ }^{199} \mathrm{Cs}$ N.M.R. experiments on solutions of the alkali salts of biphenyl and fluorenone in various ethers are presented. The contributions of the Fermi contact interaction and the chemical shielding to the shift of the N.M.R. signal are discussed. Sign and magnitude of the alkali hyperfine splitting constants are derived from the experimentally measured shifts and their variation with temperature and solvent are analysed.
\end{abstract}

\section{INTRODUCTION}

In the past few years radical ion pairs have been studied successfully by means of alkali N.M.R. experiments [1-4]. The direction of the shift of the alkali N.M.R. signal furnished important information about the sign of the unpaired spin density at the metal nucleus and it was shown that a study of linewidths and shifts could give interesting information about the structure of the ion pairs. In the light of the results obtained attempts have been made to describe the spin density at the metal nucleus and the bonding between alkali ion and radical anion in terms of the MO theory [5]. For details the reader is referred to recent review articles [6].

Here we wish to report on alkali N.M.R. experiments of the alkali salts of biphenyl and fluorenone in solutions of various ethers ; signals of the cations of ${ }^{6} \mathrm{Li},{ }^{7} \mathrm{Li},{ }^{23} \mathrm{Na},{ }^{39} \mathrm{~K},{ }^{85} \mathrm{Rb},{ }^{87} \mathrm{Rb}$ and ${ }^{133} \mathrm{Cs}$ were observed and the variation of their shifts with solvent and temperature will be analysed. A linewidth analysis will be given in a subsequent article. A preliminary account of this work was presented elsewhere [6].

Since, contrary to E.S.R. experiments, resonance positions in the N.M.R. spectrum of a radical ion pair are not only determined by the Fermi contact interaction, but also by the chemical shielding of the nucleus being investigated, it is important to establish the relative magnitude of these two terms. This will be discussed in $\S 3$. In the discussion of the experimental results in $\S 5$ it will be shown that the variation of the hyperfine coupling constants with temperature and solvent can be completely accounted for on the basis of the existing theories about ion pairing in solutions $[6,7]$. That an increase in temperature often favours a transition from solvent separated ('loose') to contact ('tight') ion 
pairs is clearly reflected in our results. Also the different abilities of the various solvents to solvate the cations become manifest in the alkali resonance positions.

\section{EXPERIMENTAL}

Sample preparation and N.M.R. apparatus have been described elsewhere $[8,4]$. Shifts were measured with respect to the signals of moderately concentrated aqueous solutions of the alkali halides and corrections were applied to obtain the shifts with respect to an infinitely diluted reference solution [9]. In this way all shifts refer to the N.M.R. signal of the completely hydrated alkali nucleus at room temperature. For $\mathrm{Li}, \mathrm{Na}$ and $\mathrm{K}$ this correction appeared to be negligible [9]. Sample concentrations were determined by weighing the amount of hydrocarbon to be used and by measuring the solvent shift at the end of the reduction [10]. The concentration determined according to the latter method always turned out to be smaller than the initial concentration determined by weighing the sample [10].

The abbreviations THP, MTHF, THF, DME, Dg, Tg, Ttg, Bp and Fl will be used throughout this article to denote the solvents tetrahydropyran, 2-methyltetrahydrofuran, tetrahydrofuran, dimethoxyethane, diglyme, triglyme and tetraglyme, and the compounds biphenyl and fluorenone, respectively. The formulae of DME, Dg, Tg and Ttg are presented by

$$
\mathrm{CH}_{3} \mathrm{O}\left[\mathrm{CH}_{2} \mathrm{CH}_{2} \mathrm{O}\right]_{n} \mathrm{CH}_{3}
$$

with $n=1,2,3$, and 4 , respectively.

In addition to the systems listed in table 2 the following systems were studied by alkali N.M.R.: ${ }^{6} \mathrm{LiBp}$ in THP, MTHF and THF and ${ }^{85} \mathrm{RbBp}$ in DME and Dg. Reduction of $\mathrm{Bp}$ in THP, MTHF and THF with $\mathrm{K}, \mathrm{Rb}$, or Cs resulted in decomposition and/or the formation of dark coloured deposits. Also reduction of $\mathrm{Bp}$ in DME with Cs proved to be difficult. For these reasons no results are reported for these systems. Also the $\mathrm{S} / \mathrm{N}$ ratio in the ${ }^{85} \mathrm{Rb}$ experiments on $\mathrm{RbBp}$ in $\mathrm{Tg}$ and $\mathrm{Ttg}$ and in the ${ }^{87} \mathrm{Rb}$ experiments on $\mathrm{RbBp}$ in $\mathrm{Ttg}$ was insufficient to get reliable data. Partial reduction of Bp with Na occurred in THP and MTHF in accordance with results of Shatenshtein et al. [11].

${ }^{6} \mathrm{Li}$ experiments were performed on ${ }^{6} \mathrm{Li}$ enriched samples. For the reduction of all other samples alkali metals were used with the natural isotopic composition.

\section{Contact ShifT versus Chemical SHielding}

The shift, $\delta_{\mathrm{c}}{ }^{\text {exp }}$, of the N.M.R. signal of an alkali nucleus in a paramagnetic ion pair measured with respect to the resonance signal of the completely hydrated alkali ion at room temperature can be presented by [12] :

$$
\delta_{\mathrm{o}}^{\exp }=\frac{B-B_{0}}{B_{0}}=\delta_{\mathrm{o}}{ }^{0}+\Delta \sigma,
$$

in which $B$ and $B_{0}$ are the fields at which resonance occurs in the ion pair and the hydrated alkali ion, respectively, and $\Delta \sigma$ is given by

$$
\Delta \sigma \equiv \sigma_{\mathrm{ip}}-\sigma_{\mathrm{aq}},
$$


in which $\sigma_{\mathrm{ip}}$ and $\sigma_{\mathrm{aq}}$ are the screening constants representing the chemical shielding of the nucleus in the ion pair and in the hydrated ion, respectively. $\delta_{\mathrm{c}}{ }^{0}$ is the contact shift and for a doublet radical it is given by [13] :

$$
\delta_{\mathrm{c}}{ }^{0}=-\frac{\left|\mu_{B}\right|}{\mu_{N}} \frac{A}{4 k T}
$$

in which $A$ represents the Fermi contact interaction constant in energy units and $\mu_{B}$ and $\mu_{N}$ are the gyromagnetic ratios of electron and nucleus, respectively. $A$ is related to the hyperfine splitting constant (hfsc), a, known from E.S.R. experiments, by

$$
A=\left|\mu_{B}\right| \hbar . a .
$$

Since the magnitude of $\Delta \sigma$ is hard to establish, $\Delta \sigma$ was dropped from equation (1) and the equation

$$
\delta_{\mathrm{c}}{ }^{\mathrm{exp}}=\delta_{\mathrm{c}}{ }^{0},
$$

was used instead.

The neglect of $\Delta \sigma$ introduces a systematic error in the determination of $\delta_{\mathrm{c}}{ }^{0}$ which in the case of ${ }^{1} \mathrm{H}$ or ${ }^{2} \mathrm{H}$ N.M.R. experiments on radicals will usually be small [12], but which may become appreciable in the case of alkali N.M.R. experiments. Since errors in $\delta_{\mathrm{o}}{ }^{\mathrm{o}}$ directly affect the value of the hfsc derived from experiment (equation (3)) the parameter $\Delta \sigma$ will be considered in more detail.

According to Ramsey [14] the screening constant is the sum of a diamagnetic part, $\sigma_{\mathrm{d}}$, which accounts for the diamagnetic current induced in the electron cloud around the nucleus by the magnetic field, and a paramagnetic part, $\sigma_{p}$, which originates from the first order mixing of excited states into the ground state by the magnetic field resulting in a non-zero electronic angular momentum. If the shielding constants, $\sigma$, of the alkali nuclei are taken relative to those of the free alkali ions in the gas phase, then $\left|\sigma_{\mathrm{a}}\right| \ll\left|\sigma_{\mathrm{p}}\right|$ so that $\sigma \approx \sigma_{\mathrm{p}} \leqslant 0$. The (negative) paramagnetic contribution, $\sigma_{\mathrm{p}}$, is determined by interactions between the electron cloud of the alkali ion and its surroundings. One may distinguish between $(a)$ van der Waals interactions, $(b)$ electrostatic polarization of the core of the alkali ion and $(c)$ overlap between valence orbitals of the cation and orbitals of electrons in the solvent shell of the alkali ion $[15,16]$. The contribution of (a) to $\sigma_{\mathrm{p}}$ is negligible [16], the contribution of $(b)$ becomes important only when small strongly polarizing cations or anions are present in the solution [16]. Usually the third term is considered to be the most important. Variation in $\sigma$ may in that case arise from variations in structure and composition of the solvent shell of the alkali ion and consequently concentration, temperature, solvent and anion may affect $\sigma$.

In order to obtain an idea about the range over which $\sigma$ may vary the largest and the smallest values of $\sigma-\sigma_{\mathrm{aq}}$ which could be found in the literature are listed in table 1 for various alkali ions. Also, measured or estimated values of $\sigma_{\mathrm{aq}}$ are given. In nearly all cases reported in the literature the variation of $\sigma$ resulted from a variation of anion and solvent ; the influence of the temperature has not been investigated extensively [17]. The most thoroughly studied cases seem to be the solutions of sodium salts ; the quoted range for $\mathrm{Na}$ is therefore probably the relatively largest one. (Interestingly, since $\sigma \leqslant 0$ it follows that 


\begin{tabular}{|c|c|c|c|c|c|}
\hline & \multicolumn{2}{|c|}{$\left(\sigma-\sigma_{\mathrm{aq}}\right) \times 10^{6}$} & \multirow[b]{2}{*}{ Reference } & \multirow[b]{2}{*}{$\sigma_{\mathrm{aq}} \times 10^{6}$} & \multirow[b]{2}{*}{ Reference } \\
\hline & minim. & $\max$ & & & \\
\hline${ }^{7} \mathrm{Li}$ & -3 & +6 & (a) & & \\
\hline${ }^{23} \mathrm{Na}$ & -17 & +16 & (b) & -10 & (d) \\
\hline${ }^{38} \mathrm{~K}$ & & & & -40 & (d) \\
\hline${ }^{85} \mathrm{Rb}$ & & & & -200 & (e) \\
\hline${ }^{189} \mathrm{Cs}$ & -57 & +63 & (c) & -340 & (e) \\
\hline
\end{tabular}

(a) Maciel, G. E., Hancock, J. K., Lafferty, L. F., Mueller, P. A., and Musker, W. K., 1966, Inorg. Chem., 5, 554 ; Dixon, J. A., Gwinner, P. A., and Lini, D. C., 1965, 7. Am. Chem. Soc., 87, 1379.

(b) Ehrlich, R. H., and Popov, A. I., 1971, F. Am. Chem. Soc., 93, 5620 ; Herlem, M., and Popov, A. I., 1972, ibid., 94, 1431 ; BLOoR, E. G., and KIDD, R. G., 1968, Can. $\mathcal{F}$. Chem., 46, 3425 .

(c) Ref. [16].

(d) Estimated values : Ref. [18].

(e) Lutz, O., 1968, Z. Naturf., 23a, 1202.

Table 1. Minimum and maximum values of $\left(\sigma-\sigma_{\mathrm{aq}}\right)$ compiled from literature data of N.M.R. experiments on aqueous and non-aqueous solutions of diamagnetic alkali salts at room temperature. Experimental or estimated values of $\sigma_{\mathrm{aq}}$ are quoted also. A positive value of $\sigma$ refers to an upfield shift.

$\sigma_{\mathrm{aq}}$ must be smaller in the algebraic sense than the smallest reported value of $\left(\sigma_{a q}-\sigma\right)$ which amounts to -16 p.p.m. (see table 1) for Na. This is smaller than the previously estimated value of -10 p.p.m. for $\mathrm{Na}$ [18]). The range quoted for $\mathrm{Cs}$ originates from a single study of solutions of $\mathrm{CsCl}$ in a large number of organic solvents [16].

The data of table 1 should be compared with those of table 2 where maximum and minimum values of $\delta_{\mathrm{c}}{ }^{\exp }$ are quoted for the various systems investigated in this study. The comparison shows that for the fluorenone salts $\Delta \sigma$ contributes only little to $\delta_{\mathrm{c}}{ }^{\text {exp }}$ so that in these cases its neglect seems to be justified. For the alkali salts of biphenyl on the other hand, especially in the case of some $\mathrm{LiBp}$ and $\mathrm{NaBp}$ solutions, the range of values of $\delta_{c}{ }^{\exp }$ is comparable to the range of values of $\sigma-\sigma_{\mathrm{aq}}$ and the varation in $\delta_{\mathrm{e}}{ }^{\mathrm{exp}}$ might therefore originate from a variation in $\delta_{\mathrm{c}}{ }^{0}$ as well as in $\Delta \sigma$. Some information about whether $\delta_{\mathrm{c}}{ }^{0}$ or $\Delta \sigma$ dominates $\delta_{\mathrm{c}}{ }^{\mathrm{exp}}$, and particularly what determines the variation of $\delta_{\mathrm{e}}{ }^{\mathrm{exp}}$ with temperature can be obtained as follows.

In the first place it should be noted that the spread in $\left(\sigma-\sigma_{\mathrm{aq}}\right)$ in table 1 almost exclusively derives from variation in solvent or anion while the variations in $\delta_{\mathrm{e}}{ }_{\mathrm{e}}^{\exp }$ for a particular alkali Bp solution derive solely from changes in temperature. ${ }^{23} \mathrm{Na}$ N.M.R. studies on solutions of sodium tetrahydroborate $\left(\mathrm{NaBH}_{4}\right)$ and tetraphenylborate $\left(\mathrm{NaB}(\mathrm{Ph})_{4}\right)$ have demonstrated that the variation of $\sigma-\sigma_{\mathrm{aq}}$ as a function of the same solvents as used in the present study are minor [17]. It also appeared that $\sigma-\sigma_{\mathrm{aq}}$ was positive and either remained constant or increased with temperature, which in our case would result in a negative temperature coefficient of the hfsc (see equation (3)). This is contrary to most experimental findings (see for instance the results for the LiBp and $\mathrm{NaBp}$ solutions shown in figures $1,2,3$ and 4). Our conclusion therefore is that, 


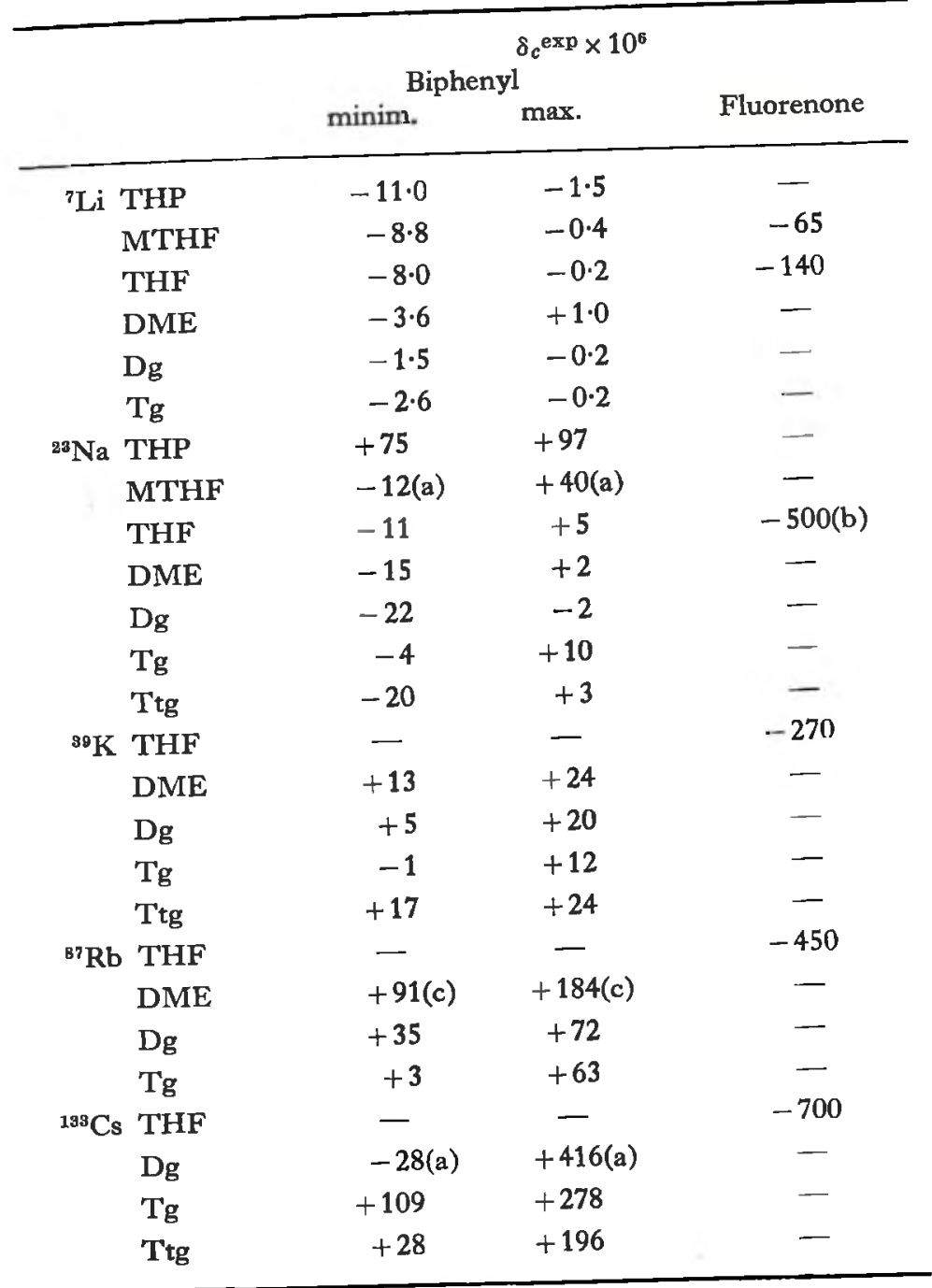
(a) See Ref. [2].
(b) See Ref. [1].
(c) See Ref. [1].

Table 2. Maximum and minimum values of $\delta_{c}{ }^{\exp } \times 10^{6}$ measured for the alkali biphenyl and alkali fluorenone salts in various solvents. The variation in the shift for a particular alkali biphenyl solution arises from variations in the temperature. For the fluorenone salts no strong variation of the shifts with the temperature was observed and only one value of $\delta_{c} e^{\exp }$ is quoted. A positive value of $\delta_{c} c^{\exp }$ refers to an upfield shift. Shifts are given with respect to the alkali N.M.R. signals of infinitely dilute aqueous alkali halide solutions at room temperature.

although for the alkali biphenyl salts in ethereal solvents the contribution of $\Delta \sigma$ to $\delta_{\mathrm{c}}{ }^{\exp }$ may be non-negligible, in which case its neglect will lead to systematically too low values of the hfsc, the variations of the hfsc with temperature are only slightly affected by $\Delta \sigma$. A linewidth analysis as well as a comparison with E.S.R. data may provide an additional check on this conclusion (see $\$ 5$ ). 


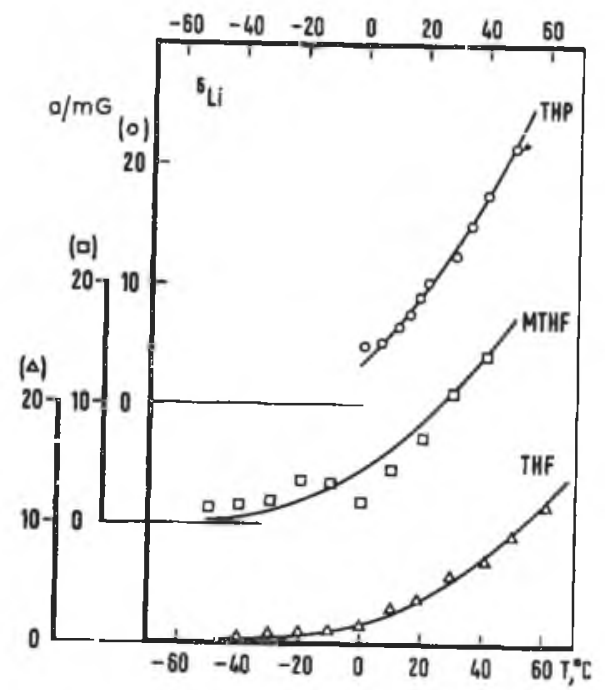

Figure 1. ${ }^{6} \mathrm{Li} \mathrm{hfsc}$ in $\mathrm{mG}\left(\mathrm{G}=10^{-4} \mathrm{~T}\right)$ as a function of the temperature for solutions of ${ }^{6} \mathrm{LiBp}$ in THP $(0.87 \mathrm{M}), \mathrm{MTHF}(0.84 \mathrm{M})$ and THF $(0.97 \mathrm{M})$.

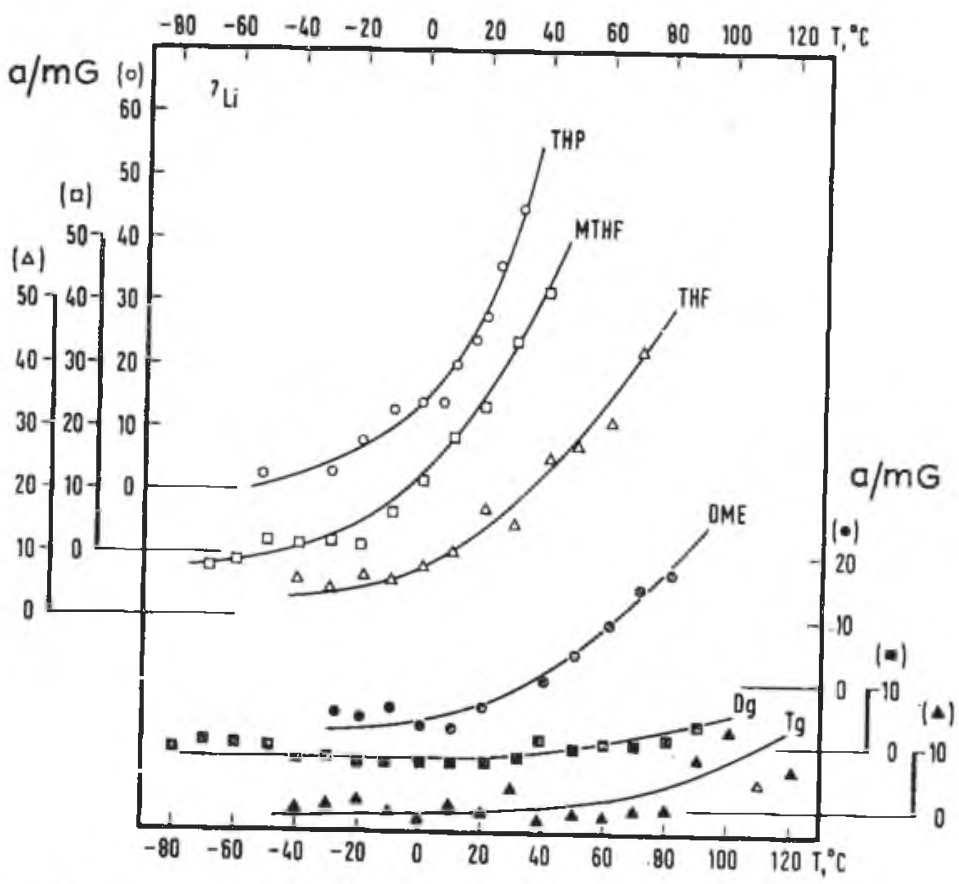

Figure 2. ${ }^{7} \mathrm{Li}$ hfsc in $\mathrm{mG}$, as a function of the temperature for solutions of $\mathrm{LiBp}$ in T.H.P. (1.05), MTHF (1.15 M), THF (1.21 M), DME (0.94), Dg (0.73 M) and Tg (0.76 M). 


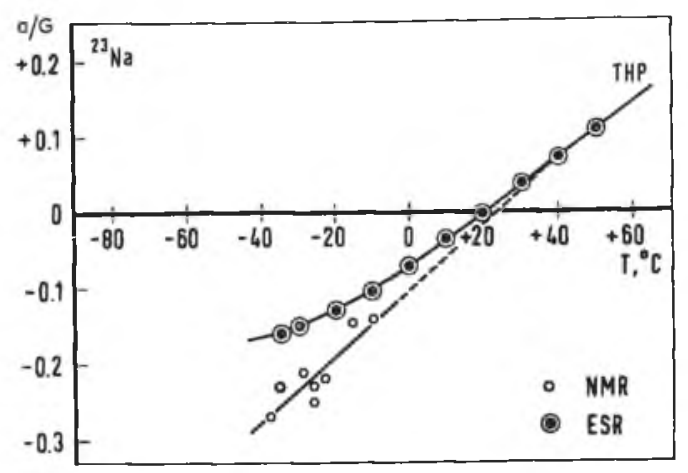

Figure 3. ${ }^{2 s} \mathrm{Na} \mathrm{hfsc}$ in $\mathrm{G}$ as a function of the temperature for solutions of $\mathrm{NaBp}$ in THP (1.15 M). E.S.R. data taken from: GracefFa, P., and Tuttle, JR., T. R., 1969, F. chem. Phys., 5, 1908.

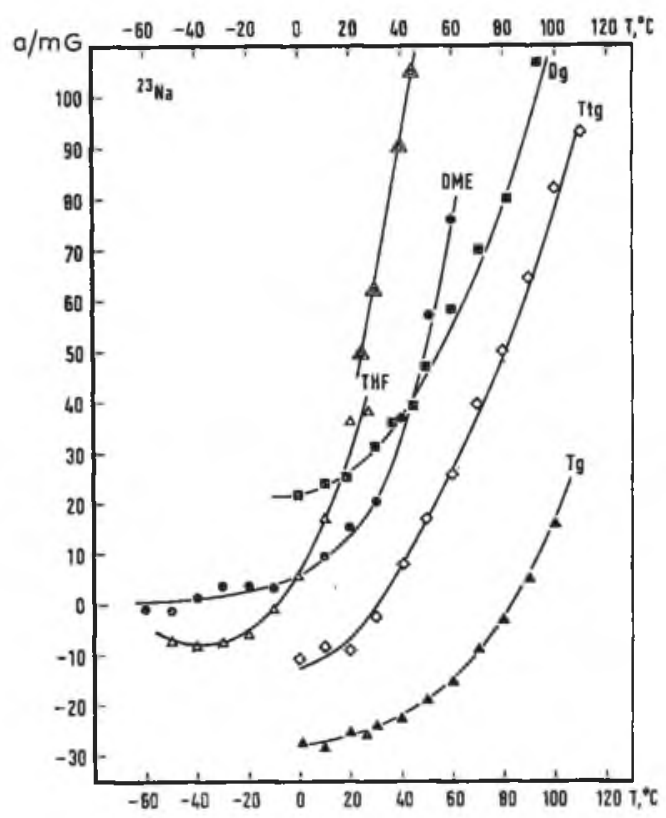

Figure 4. ${ }^{29} \mathrm{Na}$ hfsc in $\mathrm{mG}$ as a function of the temperature for solutions of $\mathrm{NaBp}$ in THF (1.94 M), DME (1.0 M), Dg (1.7 M), Tg (1.0 M) and $\mathrm{Ttg}(0.77 \mathrm{M})$. Double triangles represent E.S.R. data for $\mathrm{NaBp}$ in T.H.F. (TutrLe, JR., T. R., private communication).

\section{REsults}

From the measured shifts of the alkali N.M.R. signals the hfsc have been calculated by means of the equations (3), (4) and (5). The data have been plotted as a function of the temperature in the figures 1-8 for the biphenyl salts and in figure 9 for the fluorenone salts. In the legends to the figures the initial concentrations have been indicated. Data for $\mathrm{NaBp}$ in MTHF have been published elsewhere [2] and are not presented here. E.S.R. data for the 

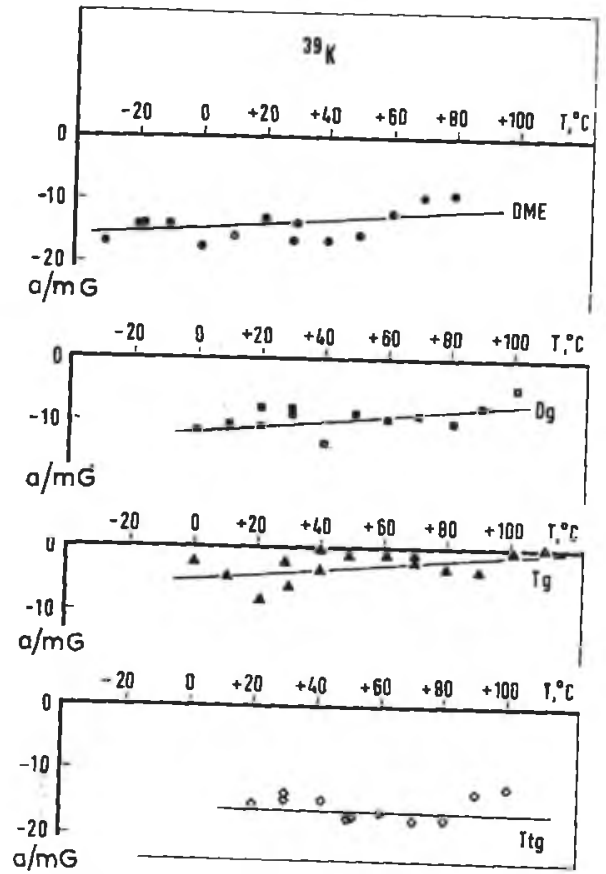

Figure 5. ${ }^{99} \mathrm{~K}$ hfsc in $\mathrm{mG}$ as a function of the temperature for solutions of $\mathrm{KBp}$ in DME (1.21 M), Dg (0.74 M), $\mathrm{Tg}(1.51 \mathrm{M})$ and $\mathrm{Ttg}(0.77 \mathrm{M})$.

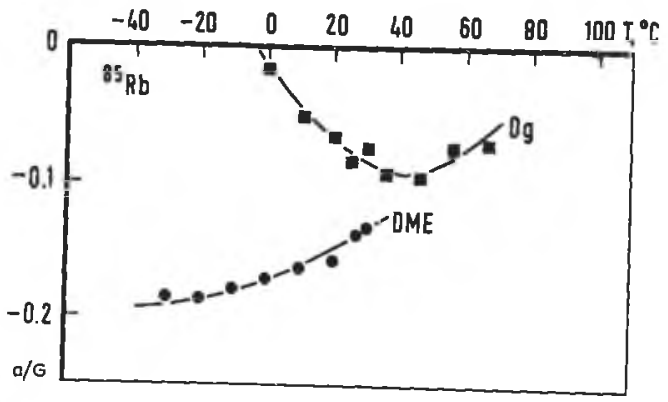

Figure 6. ${ }^{85} \mathrm{Rb}$ hfsc in $\mathrm{G}$ as a function of the temperature for solutions of $\mathrm{RbBp}$ in DME $(1.50 \mathrm{M})$ and $\mathrm{Dg}(1.25 \mathrm{M})$. Data for the DME solution taken from ref. [1].

biphenyl salts, when available, have been plotted also. For E.S.R. data on the fluorenone anion the reader is referred to the work of Hirota [19].

In solutions of the $\mathrm{Li}$ and $\mathrm{Cs}$ salts of fluorenone sometimes a relatively sharp signal with $\delta_{\mathrm{o}}{ }^{\mathrm{exp}} \approx 0$ was observed in addition to the signal of the paramagnetic ion pair. We attribute this signal to diamagnetic dimers [20] and refer for further details to an earlier discussion [12].

As a check on the experimental procedure the ratios of the ${ }^{6} \mathrm{Li}$ and ${ }^{7} \mathrm{Li}$ hfsc measured in various $\mathrm{LiBp}$ solutions were calculated. In all cases studied the ratio agreed within experimental error with the theoretical ratio of the gyromagnetic constants. Similar results were obtained for the ${ }^{85} \mathrm{Rb}$ and ${ }^{87} \mathrm{Rb}$ hfsc. 


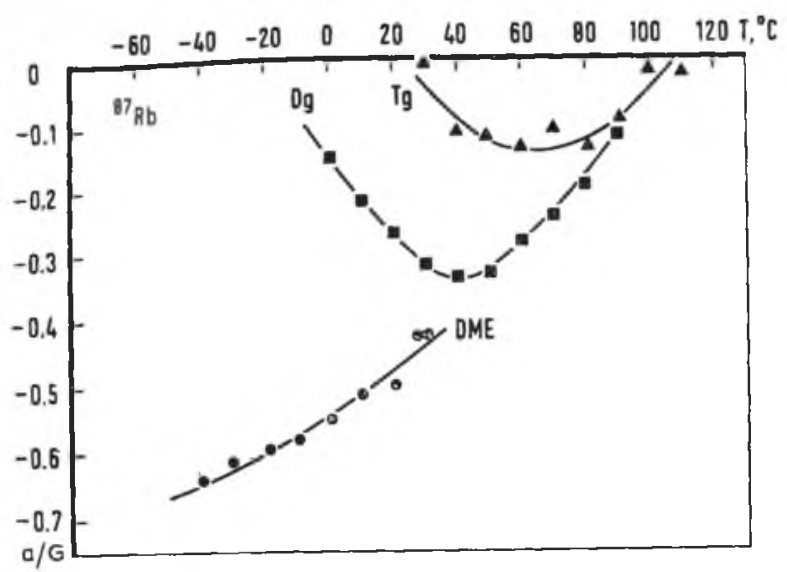

Figure 7. ${ }^{87} \mathrm{Rb}$ hfsc in $\mathrm{G}$ as a function of the temperature for solutions of $\mathrm{RbBp}$ in DME (1.50 M), Dg (1.25 M) and $\mathrm{Tg}(0.90 \mathrm{M})$. Data for the DME solution taken from ref. [1].

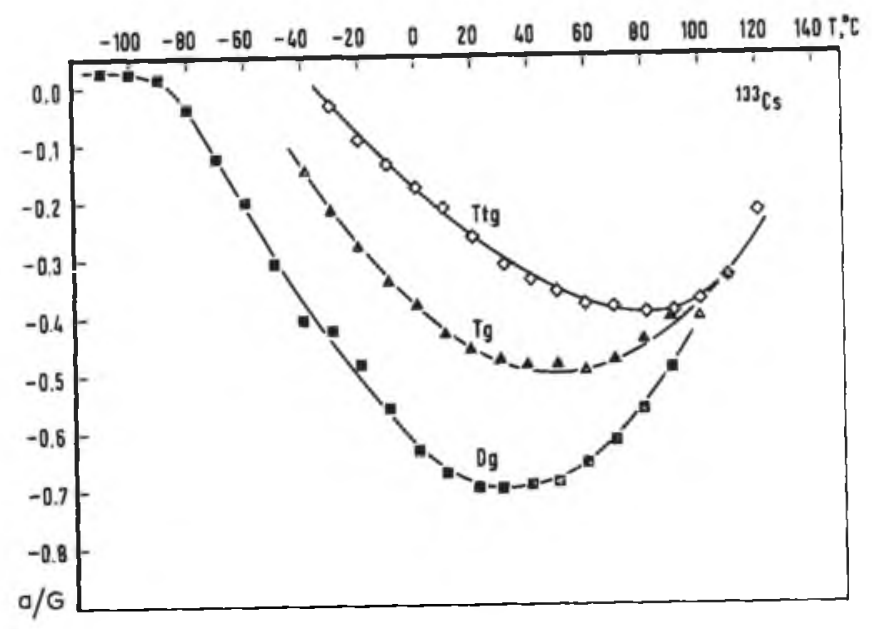

Figure 8. ${ }^{1 a a} \mathrm{Cs} h f s c$ in $\mathrm{G}$ as a function of the temperature for solutions of $\mathrm{CsBp}$ in $\mathrm{Dg}$ (1.44), Tg $(0.87 \mathrm{M})$ and $\mathrm{Ttg}(0.65 \mathrm{M})$. Data for the $\mathrm{Dg}$ solution taken from ref. [2].

\section{Discussion}

The data of the biphenyl salts are discussed first. The influence of temperature and solvent on the hfsc, and the sign of the splitting constant will be considered and the N.M.R. data will be compared with E.S.R. results. Next the metal hfsc of the alkali salts of fluorenone will be dealt with. In the discussion of the temperature and solvent dependence of the metal hfsc the KBp systems are left out of consideration. The spread in the data prevents to establish a trend in the temperature dependence of the hfsc. In addition not enough ${ }^{39} \mathrm{~K}$ N.M.R. data for solutions of diamagnetic potassium salts are available to make a reliable estimate of the relative magnitudes of $\delta_{\mathbf{c}}{ }^{0}$ and $\Delta \sigma$. 


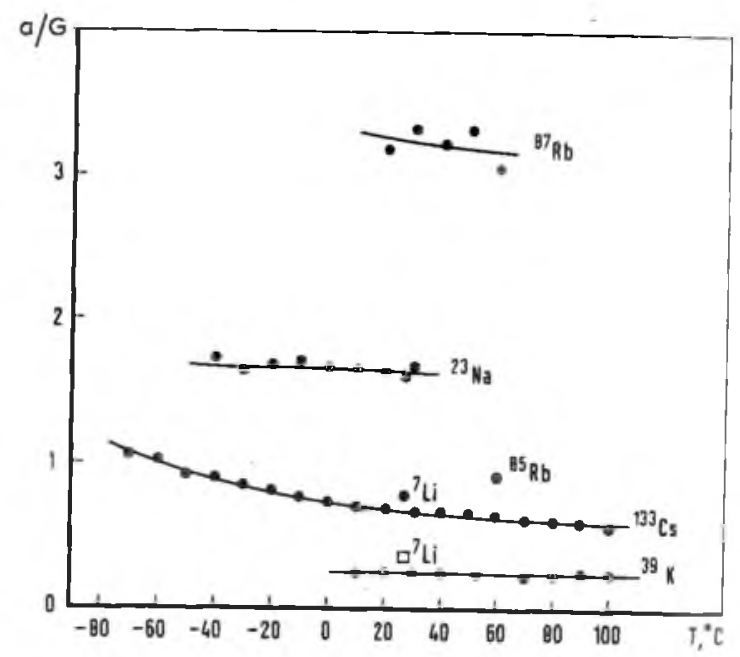

Figure 9. Alkali hfsc in $\mathrm{G}$ as a function of the temperature for solutions of ${ }^{2} \mathrm{LiFl}(1 \cdot 15 \mathrm{M})$, ${ }^{28} \mathrm{NaFl}(1.1 \mathrm{M}),{ }^{39} \mathrm{KFl}(0.77 \mathrm{M}),{ }^{85} \mathrm{RbFl}$ and ${ }^{87} \mathrm{RbFl}(1.02 \mathrm{M})$ and $\mathrm{CsFl}(0.94 \mathrm{M})$ in THF. The open quadrangle denotes the ${ }^{7} \mathrm{Li} \mathrm{hfsc}$ for $\mathrm{LiFl}$ in MTHF (1.0 M).

\subsection{Biphenyl}

(i) Temperature dependence. For the solutions of $\mathrm{NaBp}$ in THP (figure 3) and in MTHF [2] and of RbBp in DME (figures 6 and 7) a monotonous increase in the hfsc with temperature is observed. Inspection of table 1 and 2 shows that in these systems the Fermi contact interaction probably plays a dominant role in determining the shift. Our observations can therefore be explained if we assume that over the investigated temperature range in these systems contact ion pairs exist in which the average position of the alkali ion with respect to the radical is temperature dependent. Since the metal spin density depends on the position of the alkali ion [5] the hfsc changes with temperature.

The $a$ versus T-plots for all the other alkali Bp-systems exhibit at high temperature a positive slope. In nearly all cases the slope is large enough to render it likely that also in these systems the Fermi contact interaction dominates the variation in the hfsc in accordance with the conclusion put forward at the end of $\S 3$. On the other hand, towards low temperatures nearly all the hfsc in these systems decrease in absolute value or level off to a constant, often zero, value. Apparently the average solvation of the cation increases on going to lower temperatures causing a decrease of the Fermi contact interaction so that finally at very low temperatures $\Delta \sigma$ dominates the shift. The latter effect is visible in the case of the $\mathrm{LiBp}$ and $\mathrm{NaBp}$ solutions, where at low temperatures a residual small variation of the hfsc is observed as a function of the solvent (figures 2, 4). Especially the effect of $\mathrm{Tg}$ and $\mathrm{Ttg}$ on $\Delta \sigma$ has been noted previously [17] (see figure 4) and probably points to a special ion pair structure. This will be discussed more fully in a subsequent paper dealing with a line width analysis, in which it is shown that the increase in alkali line width going to higher temperatures can be ascribed to enhanced anisotropic dipolar interaction. These observations suggest a structure of the ion pair, in which the alkali ion is surrounded by the long glyme molecules in a pseudo-crown ether fashion, thus creating space for the $\mathrm{Bp}$ anion to approach the cation more closely. Also 
the fact that easily single crystals can be formed from $\mathrm{NaBp}$ in $\mathrm{Tg}$ and $\mathrm{Ttg}$ points to a specific ion pair structure [8].

(ii) Solvent dependence. The influence of a particular solvent on the structure of an ion pair is usually related to the solvating power of the solvent towards the cation being considered [7]. The solvating power of the solvents used in this study increases in the order THP $<\mathrm{MTHF}<\mathrm{THF}<\mathrm{DME}<\mathrm{Dg}<\mathrm{Tg}<\mathrm{Ttg}$ [7]. One would expect therefore that at a properly chosen temperature one would observe for a particular alkali $\mathrm{Bp}$ salt a change in ion pair structure from contact (tight) to solvent separated (loose) going along the above mentioned series of solvents, which would result in a decrease of the absolute value of the hfsc. This is borne out by the experimental findings for the $\mathrm{Rb}$ and $\mathrm{CsBp}$ solutions (figures 6-8).

Since for most of the solutions of $\mathrm{LiBp}$ and $\mathrm{NaBp}$ there is probably a considerable contribution from $\Delta \sigma$ to $\delta_{\mathrm{a}}$ exp, while the variation in the hfsc with temperature is probably determined only by $\delta_{\mathrm{c}}{ }^{0}$ (see $\S 3$ ), the criterion for the solvating power can be better formulated in terms of the slopes of the plots : the smaller the solvating power the larger the slope at a given temperature and the lower the temperature at which a given value of the slope will be reached. In table 3 the slopes of the plots for the ${ }^{7} \mathrm{LiBp}$ and $\mathrm{NaBp}$ solutions at various temperatures are presented. The data confirm the above mentioned order of the solvents according to their solvating power : for $\mathrm{LiBp}$ the slope at $30^{\circ} \mathrm{C}$ decreases regularly from THP through $\mathrm{Tg}$ while for $\mathrm{NaBp}$ the temperature at which a particular slope is reached increases from THF through Ttg (with an unimportant irregularity at the transition from $\mathrm{Tg}$ to $\mathrm{Ttg}$ ) and even higher slopes are found at still lower temperatures in THP and MTHF.

\begin{tabular}{|c|c|c|c|c|}
\hline & \multicolumn{2}{|c|}{${ }^{7} \mathrm{Li}$ Biphenyl } & \multicolumn{2}{|c|}{${ }^{23} \mathrm{Na}$ Biphenyl } \\
\hline & Slope & Temperature $/{ }^{\circ} \mathrm{C}$ & Slope & Temperature $/{ }^{\circ} \mathrm{C}$ \\
\hline THP & 150 & +30 & 440 & $<-10$ \\
\hline MTHF & 80 & +30 & 240 (a) & $<0$ (a) \\
\hline THF & 35 & +30 & 225 & 10 \\
\hline DME & 23 & +30 & 225 & 30 \\
\hline $\mathrm{Dg}$ & 7 & +30 & 225 & 65 \\
\hline $\mathrm{Tg}$ & 5 & +30 & 225 & 90 \\
\hline Ttg & - & - & 225 & 65 \\
\hline
\end{tabular}

(a) See Ref. [2].

Table 3. Slopes in $\mathrm{mG} / 100^{\circ} \mathrm{C}$ of the plots in the figures 2,3 and 4 at various temperatures.

Finally the data in the figures 1-8 demonstrate the influence of the cation radius on the ion pair structure. One would expect that in a given solvent the salts of a particular anion will form more easily solvent separated ion pairs as the radius of the cation decreases [7]. This is in accordance with the experimental findings. For example, it appears that $\mathrm{LiBp}$ and $\mathrm{NaBp}$ form solvent separated ion pairs in DME at low temperatures whereas $\mathrm{RbBp}$ forms only contact ion pairs in this solvent. 
(iii) Sign. In the alkali $\mathrm{Bp}$ ion pairs the alkali hfsc is positive for $\mathrm{Li}$ (the negative sign of the Li hfsc at low temperatures in DME, Dg and Tg probably is caused by $\Delta \sigma$ ), for $\mathrm{Na}$ the hfsc can be positive as well as negative, depending on the solvent, and in one case even passes through zero (namely in MTHF [2]; the change in sign in THF, $\mathrm{Tg}$ and $\mathrm{Ttg}$ may arise from the effect of $\Delta \sigma$ on the shift), and for $\mathrm{Rb}$ and $\mathrm{Cs}$ the hfsc is negative. Finally, although for $\mathrm{K}$ the relative magnitudes of $\delta_{\mathrm{c}}{ }^{0}$ and $\Delta \sigma$ and therefore the sign of $\delta_{\mathrm{c}}{ }^{0}$ is not known the $\mathrm{K}$ hfsc are close to zero. These observations confirm a trend noted before $[4,8,21]$, namely that with $\pi$-electron radicals like biphenyl-, anthracene ${ }^{-}$and naphthalene- the unpaired spin density at the metal nucleus shows the tendency to become negative with increasing cationic radius.

According to recent theories $[5(b)]$ the total unpaired spin density at the nucleus, $\rho$, is the sum of a positive zero order contribution $\rho_{0}$ and a first order contribution $\rho_{1}$, which for the radical anions of the above mentioned type often will be negative. $\rho_{0}$ and $\rho_{1}$ depend on the overlap of the valence $s$-orbital of the metal with the first anti-bonding MO and the bonding MO's of the radical, respectively. Since overlap with the two types of MO may depend differently on the position of the cation with respect to the radical, $\rho_{0}$ and $\rho_{1}$ may vary differently with the average cation position. In this way a variation in the average position of the cation with temperature could result in a change of sign of the alkali hfsc. Further it has been demonstrated $[5(b)]$ that with an increase in the distance between cation and anion $\rho_{0}$ in general will fall off more rapidly than $\rho_{1}$. This could explain the above mentioned trend in the hfsc.

(iv) Comparison with E.S.R. data. The available E.S.R. data (NaBp in THP (figure 3), in MTHF [2] and in THF (figure 4)) demonstrate that the N.M.R. hfsc are equal to or smaller than the E.S.R. hfsc. The difference probably originates from the neglect of $\Delta \sigma$ in equation (1). As argued in $\S 3$, in the solvents used in this study this term is probably positive. Its neglect will result in values of $\left|\delta_{\mathrm{o}}{ }^{0}\right|$, which are too large if $\delta_{\mathrm{o}}$ exp is positive and too small if $\delta_{\mathrm{c}}{ }^{\exp }$ is negative. According to equation (3) this means that positive N.M.R. hfsc are always smaller than the corresponding E.S.R. hfsc, whereas negative N.M.R. hfsc are larger in absolute value than the corresponding E.S.R. hfsc. Hence in plots of $a$ vs $T$ the N.M.R. line will lie lower than the E.S.R. line, as is actually observed (see figures 3, 4 and references [2] and [6]). However, the similar slopes of the two curves confirm the conclusion put forward in $\$ 3$, that the observed variation in the hfsc with the temperature is governed by the variations in the Fermi contact interaction. Similar observations were made for the alkali salts of naphthalene [4].

\subsection{Fluorenone}

Contrary to the alkali $B p$ salts the alkali hfsc for fluorenone are not very much temperature dependent (see figure (9)). Apparently the cations are attracted strongly enough by the radical to prevent complete solvation of the alkali ions at low temperatures. Further for all the alkali ions the hfsc are large and positive, indicating that the contribution from $\rho_{0}$ to the total spin density at the metal nucleus is considerable. It is concluded that the alkali ions in the fluorenone ion pairs are close to a site with appreciable charge and spin density, probably the oxygen atom. This is in accordance with E.S.R. findings [22]. 
The difference between E.S.R. [19] and N.M.R. hfsc is appreciable and probably points to a difference in ion pair structure $[20,22]$. The E.S.R. hfsc refer to monomer ion pairs while the N.M.R. experiments were performed on concentrated solutions in which most probably the ion pairs are present in the form of dimers or higher aggregates [20,22].

\section{Conclusion}

Alkali N.M.R. experiments on alkali radical ion pairs can be an important supplement to E.S.R. experiments, particularly with regard to the sign of the metal hfsc. When the contact shift is small, the method suffers from the drawback that the contribution from $\Delta \sigma$ to the experimentally measured shift may become appreciable and that its magnitude is difficult to establish. The interpretation of the N.M.R. data, however, can be simplified if the temperature dependence of the shift is taken into consideration.

It is pointed out that the concentration dependence of the shifts has not been studied in detail. In so far as the concentration affects the ion pair structure (as in the case of the fluorenone salts, for instance) both $\delta_{\mathrm{c}}{ }^{0}$ and $\Delta \sigma$ will depend on the concentration. In addition clustering of ion pairs may affect $\Delta \sigma$, probably more than $\delta_{0}{ }^{\circ}$. Under unfavourable circumstances these effects may complicate the interpretation of the N.M.R. data. On the other hand, since radical ion pairs are not accessible for study by E.S.R. in the high concentration range, N.M.R. may offer advantages over E.S.R. techniques in this respect.

The authors thank Dr. B. M. P. Hendriks and Mr. A. A. K. Klaassen for skilful experimental assistance during the measurements. Valuable discussions with Dr. H. van Willigen during the early stages of this work are gratefully acknowledged. Mr. L. C. E. J. M. ten Horn is thanked for the design and the construction of the N.M.R. frequency stabilizers. The present investigations have been carried out under the auspices of the Netherlands Foundation of Chemical Research (SON) and with the aid of the Netherlands Organization for the Advancement of Pure Research (ZWO).

\section{REFERENCES}

[1] Canters, G. W., De Boer, E., Hendriks, B. M. P., and Van Willigen, H., 1968, Chem. Phys. Lett., 1, 627.

[2] Canters, G. W., De Boer, E., Hendriks, B. M. P., and Klaassen, A. A. K., 1969, Colloque Ampère, 15, 242.

[3] Takeshita, T., and Hirota, N., 1969, Chem. Phys. Lett., 4, 369.

[4] Hendriks, B. M. P., Canters, G. W., Corvaja, C., De Boer, J. W. M., and De Boer, E., 1971, Molec. Phys., 20, 193.

[5] (a) Goldberg, I. B., and Bolton, J. R., 1970, F. phys. Chem., 74, 1965. (b) Canters, G. W., Corvaja C., and De Boer, E., 1971, F. chem. Phys., 54, 3026. (c) Pasimeni, L., Brustolon, M., and Corvaja, C., 1972, 7. chem. Soc., Farad. Transacts. II, 68, 223.

[6] Ions and ion pairs in organic reactions, Volume 1, Ed., M. SzwARC, Wiley-Interscience, New York, 1972 ; Szwarc, M., 1968, Carbanions, Living Polymers and Electron Transfer Processes, Interscience, New York.

[7] SmID, J., 1972, Angew. Chemie, 84, 127.

[8] Canters, G. W., 1969, Thesis, University of Nijmegen, Nijmegen, The Netherlands. 
[9] Devereil, C., and Richards, R. E., 1966, Molec. Phys., 10, 551 ; AkrtT, J. W., and Downs, A. J., 1966, Chem. Commun., 222.

[10] De Boer, E., Grotens, A. M., and Smid, J., 1970, F. Am. chem. Soc., 92, 4742.

[11] Shatenshtein, A. I., and Petrov, E. S., 1967, Russ. Chem. Revs., 36, 100.

[12] Canters, G. W., Hendriks, B. M. P., De Boer, J. W. M., and De Boer, E., 1973, Molec. Phys., 25, 1135.

[13] McConnell, H. M., and Chesnut, D. B., 1958, f. chem. Phys., 28, 107.

[14] See for instance: Carrington, A., and McLachlan, A. D., 1967, Introduction to Magnetic Resonance, Harper \& Row, New York.

[15] Deverell, C., 1969, Progress in NMR Spectroscopy, Vol. 4 (London: Pergamon Press), p. 235.

[16] Halliday, J. D., Richards, R. E., and Sharp, R. R., 1969, Proc. R. Soc. A, 313, 45.

[17] Canters, G. W., 1972, F. Am. chem. Soc., 94, 5230.

[18] Deverel. C., 1969, Molec. Phys., 16, 491.

[19] Hirota, N., 1967, Ұ. Am. chem. Soc., 89, 32

[20] Hirota, N., and Weissman, S. I., 1964, Ұ. Am. chem. Soc., 86, 2538.

[21] Herold, B. J., Neiva Correia, A. F., and dos Santos Veiga, J., 1965, $\mathscr{F}$. Am. chem. Soc., 87, 266 ; Hrrota, N., 1968, Ibid., 90, 3603 ; Dodson, C. L., and Reddoch, A. H., 1968, F. chem. Phys., 48, 3226; Warhurst, E., and Wilde, A. M., 1969, Trans. Faraday Soc., 65, 1413.

[22] Nakamura, K., and Hirota, N., 1969, Chem. Phys. Lett., 3, 134, 137. 